\title{
Electrochemical Behavior of Cobaltocene in Ionic Liquids
}

\author{
Andrzej Lewandowski · Lukasz Waligora • Maciej Galinski
}

Received: 11 March 2011/Accepted: 6 April 2012/Published online: 2 February 2013

(C) The Author(s) 2013. This article is published with open access at Springerlink.com

\begin{abstract}
The electrochemical behavior of cobaltocenium has been studied in a number of room temperature aprotic ionic liquids. Well defined, diffusion controlled, anodic and cathodic peaks were found for the $\mathrm{Cc}^{+} / \mathrm{Cc}$ (cobaltocenium/cobaltocene) reduction/oxidation on gold, platinum and glassy carbon electrodes. Values of the peak separation parameters suggest quasireversibility or even irreversibility for the redox process. The difference between the ferrocene/ferrocenium and cobaltocenium/cobaltocene couples has been evaluated as equal to $(1.350 \pm 0.020) \mathrm{V}$. Values of the cobaltocenium $\left(\mathrm{Cc}^{+}\right)$diffusion coefficients $D$ have been calculated on the basis of the Randles-Sevcik equation.
\end{abstract}

Keywords Ionic liquid · Reference redox couple $\cdot$ Cobaltocenium

\section{Introduction}

Room temperature ionic liquids [RTILs] may be used as solvents in many applications, as described in references [1-6], including electrochemistry [7-10]. In the latter case a reference system is necessary to compare potentials measured in different solvent-free RTILs. Different reference systems have been proposed, including pseudo-reference electrodes (metal immersed in an electrolyte, usually Pt or Ag), according to references $[11,12]$. In such a case, the surface of the pseudo-reference system must be much larger in comparison to the working electrode; consequently, the current density at the reference is much lower. Half-cells based on the $\mathrm{AglAg}{ }^{+}$or $\mathrm{Ag} \mid \mathrm{AgCllCl}^{-}$systems (where a salt, being a source of $\mathrm{Ag}^{+}$or $\mathrm{Cl}^{-}$ions, is dissolved in a mixture of acetonitrile with ionic liquid) have also been recommended as references for RTILs [13-15] and also for high temperature ILs (LiCl$\mathrm{KCl}-\mathrm{CaCl}_{2}$ ) [16]. Silver wire, immersed in a solution of silver(I) salt and cryptand 222 in molecular liquids [17] and ionic liquids [18], has been proposed as a stable reference system.

A. Lewandowski $(\bowtie) \cdot$ L. Waligora $\cdot$ M. Galinski

Faculty of Chemical Technology, Poznań University of Technology, 60965 Poznań, Poland

e-mail: andrzej.lewandowski@put.poznan.pl 
Finally, electrode potentials may be expressed versus an inner reference organometallic redox system, which consists of a large cation and its reduced form. The oxidized and reduced forms, both of large radius, may be assumed to be solvated similarly in different solvents. This leads directly to the assumption that redox potentials of such couples should be comparable in different solvents. Consequently, a given redox couple may be regarded as a universal potential reference. Organometallic redox couples such as bis(biphenyl)chromium(0)/(I) $\left(\mathrm{BCrlBCr}^{+}\right)$, ferrocenelferrocenium $\left(\mathrm{FclFc}^{+}\right)$, and cobaltocenelcobaltocenium $\left(\mathrm{CclCc}^{+}\right)$have been investigated in various RTILs as described in the literature [10,19-34], including a study on the applicability of cobaltocenium reduction as a reference for ionic liquids [30] and a detailed study on the simultaneous presence of both $\left(\mathrm{CcICc}^{+}\right)$and $\left(\mathrm{FclFc}^{+}\right)$couples in RTILs [32]. The general aim of the present study was to conduct a systematic investigation of the $\mathrm{Cc} / \mathrm{Cc}^{+}$redox reference system in various aprotic ionic liquids at different electrodes.

\section{Experimental}

\subsection{Chemicals}

Bis(cyclopentadienyl) cobalt(III) hexafluorophosphate (cobaltocenium hexafluorophosphate, $\mathrm{Cc}^{+} \mathrm{PF}_{6}^{-}$, Aldrich), silver perchlorate $\left(\mathrm{AgClO}_{4}\right.$, Fluka), and cryptand 222 (4.7.13.16.21.24-hexaoxa-1.10-diazabicyclo [8.8.8] hexacosane, Merck) were used as purchased. Acetonitrile (AN, Merck) was distilled before use. Room temperature ionic liquids: $N$-methyl- $N$-propylpyrrolidinium bis(trifluoromethanesulfonyl)imide (MePrPyrrNTf ${ }_{2}$ ) (Iolitec), $\mathrm{N}$-butyl- $\mathrm{N}$-methylimidazolium triflate (BuMeImOTf) (Iolitec), $N$-butyl- $N$-methylpyrrolidinium bis(trifluoromethanesulfonyl)imide (BuMePyrrNTf 2 ) (Iolitec), $N$-butyl- $N$-methylpyrrolidinium triflate (BuMePyrrOTf) (Merck), diethylmethylsulphonium bis(trifluoromethanesulfonyl)imide ([ $\left.\mathrm{Et}_{2} \mathrm{MeS}\right]\left[\mathrm{NTf}_{2}\right]$ (Iolitec), and triethylsulphonium bis(trifluoromethanesulfonyl)imide ([Et $\left.{ }_{3} \mathrm{~S}\right]\left[\mathrm{NTf}_{2}\right]$ (Iolitec) were used as purchased. $N$-ethyl- $N$-methylimidazolium dicyanoimide $\left(\operatorname{EtMeImN}(\mathrm{CN})_{2}\right)$ was obtained according to a published procedure [35] by metathesis of EtMeImBr with $\operatorname{AgN}(C N)_{2}$ in an aqueous solution [36]. $N$-methyl- $N$-propylpiperidinium bis(trifluoromethanesulfonyl)imide $\left(\mathrm{MePrPipNTf}_{2}\right)$ was obtained according to a method described in the literature [37].

\subsubsection{Water Content and Purity}

The water content in aprotic ionic liquids, analyzed with a standard Karl-Fisher titrant (HYDRANAL ${ }^{\circledR}$ Composite $1,1 \mathrm{~mL} / 10 \mathrm{mg} \mathrm{H}_{2} \mathrm{O}$ ), was below the detection limit. All of the RTILs were colorless. The purities of ionic liquids for electrochemical purpose were analyzed with cyclic voltammetry on platinum, gold and glassy carbon working electrodes. No reduction or oxidation peaks were detected between the anodic and cathodic decomposition potentials.

\subsection{Apparatus and Procedures}

Voltammetric measurements were performed in a three-electrode arrangement. Working electrodes: Au (1.50 mm diameter, $\left.1.77 \mathrm{~mm}^{2}\right)$, Pt (1.50 mm, $\left.1.77 \mathrm{~mm}^{2}\right)$ and glassy carbon (3.00 mm diameter, $7.07 \mathrm{~mm}^{2}$ ) were disc shaped and sealed in poly(tetrafluoroethylene). 
Before measurements, the electrodes were polished with aluminum oxide paste in water $\left(\mathrm{Al}_{2} \mathrm{O}_{3}, 150\right.$ mesh, Merck) and then washed with acetone. The counter electrode was a platinum sheet $(0.5 \times 1.0 \mathrm{~cm})$. The reference electrode consisted of a silver wire immersed in a solution of $\mathrm{AgClO}_{4}\left(0.01 \mathrm{~mol} \cdot \mathrm{dm}^{-3}\right)$ and cryptand $222\left(0.1 \mathrm{~mol} \cdot \mathrm{dm}^{-3}\right)$ in acetonitrile [17]. The reference electrode compartment was separated by a glass frit from the cell containing the ionic liquid. Preparation of the solutions, weighing of the samples, and cell assembly were performed in a glove-box under a dry argon atmosphere. Tested electrolytes were deaerated with argon for $30 \mathrm{~min}$ prior to measurements. Voltammetric curves were obtained with the $\mu$ AutoLab Electrochemical System (Eco Chemie, The Netherlands) at $(25 \pm 0.1){ }^{\circ} \mathrm{C}$. The initial scan was carried out to more negative potentials (reduction of cobaltocene: $\mathrm{Cc}^{+}+\mathrm{e}^{-} \rightarrow \mathrm{Cc}^{0}$ ) followed by the reverse anodic scan (oxidation of $\mathrm{Cc}^{0}$ ). Two reduction/oxidation scans were recorded in each case. The baseline of each neat ionic liquids was measured before experiments with $\mathrm{Cc} / \mathrm{Cc}^{+}$solutions. The ohmic resistance $R$ between electrodes was determined from impedance spectra (using an ac impedance analyzer Atlas-Sollich, Poland), in the frequency range of $100 \mathrm{kHz}$ to $1 \mathrm{~Hz}$ with $10 \mathrm{mV}$ amplitude.

\section{Results and Discussion}

\subsection{Electrolyte Conductivity and IR Ohmic Drop}

The conductivity of the electrolyte influences the resistance between electrodes of the cell. In the case of ionic liquid electrolytes, the specific conductivity is typically between ca. 10 and $0.01 \mathrm{mS} \cdot \mathrm{cm}^{-1}$, according to Ref. [8], which may lead to resistances differing by three orders of magnitude. The resistance was obtained by deconvolution of impedance spectra according to an equivalent circuit, consisting of resistance $R$ in series with the Warburg impedance and charge transfer resistance, in parallel to the double layer capacity. The resistance $R$, determined from impedance spectra, was between ca. $200 \Omega\left(\mathrm{Et}_{3} \mathrm{SNTf}_{2}\right)$ and $2,400 \Omega\left(\mathrm{MePrPipNTf}_{2}\right)$. At a typical current level of $10 \mu \mathrm{A}$, the $I R$ distortion of the potential was in the range of $0.2-24 \mathrm{mV}$.

\subsection{Curves}

Figure 1 presents typical cv curves, after background current and $I R$ drop corrections, for cobaltocenium/cobaltocene $\left(\mathrm{Cc}^{+} / \mathrm{Cc}\right)$ reduction/oxidation in BuMeImOTf $\left(\left[\mathrm{CcPF}_{6}^{-}\right]=\right.$ $9.18 \mathrm{mmol} \cdot \mathrm{L}^{-1}$ ) at potential sweep rates from (2 to 200) $\mathrm{mV} \cdot \mathrm{s}^{-1}$, versus the $\mathrm{Ag} \mid \mathrm{Ag}^{+} 222$, AN reference electrode. Potentials of the peak maximum, $E_{\mathrm{pa}}$ and $E_{\mathrm{pc}}$, for $\mathrm{Cc}^{+}$cathodic reduction and $\mathrm{Cc}$ anodic oxidation were -854 and $-762 \mathrm{mV}$, respectively. Similar $\mathrm{CV}$ curves were obtained for a number of ionic liquids as solvents recorded at the three different electrodes (Pt, Au, GC). Measurements of $\Delta E_{1 / 2}\left(\mathrm{Cc}^{+} / \mathrm{Cc}^{0}\right)$ over a period of $24 \mathrm{~h}$ gave stable values within ca. $1-2 \mathrm{mV}$, indicating no significant changes in the liquid junction potential between RTILs and the reference electrode electrolyte. Differences between the cathodic and anodic peak potentials, $E_{\mathrm{pa}}-E_{\mathrm{pc}}, 1 / 2\left(E_{\mathrm{pa}}+E_{\mathrm{pc}}\right)$, and $E_{\mathrm{p}}-E_{\mathrm{p} / 2}$ values $\left(E_{\mathrm{p} / 2}\right.$ is the half-peak potential), and peak current densities $j_{\mathrm{pa}}$ and $j_{\mathrm{pc}}$, in the studied ionic liquids, are collected in Table 1 . The difference between the cathodic and anodic peak potentials, $E_{\mathrm{pa}}-E_{\mathrm{pc}}$, is $\geq 81 \mathrm{mV}$, while the value predicted by the theory for reversible processes, according to Ref. [38], is (57-60) $\mathrm{mV}$ depending on the switching potential. The 
results indicate a quasi-reversible redox process for the cobaltocenium/cobaltocene couple. A similar behavior was found for the ferrocenelferrocenium couple in a number of ionic liquids in our previous paper [34]. On the other hand, the $E_{\mathrm{p}}-E_{\mathrm{p} / 2}$ values are close to $56 \mathrm{mV}$, typical of reversible processes [38]. In some protic RTILs, the $E_{\mathrm{p}}-E_{\mathrm{p} / 2}$ values are close to the theoretical value of $56 \mathrm{mV}$, but in some cases they are higher (even as much as $70 \mathrm{mV})$. The formal potential $1 / 2\left(E_{\mathrm{pa}}+E_{\mathrm{pc}}\right)$ for the $\mathrm{Cc}^{+} / \mathrm{Cc}$ couple in aprotic ionic liquids may be approximated by the value- $(831 \pm 13) \mathrm{mV}$ (versus the $\mathrm{Ag}^{\mathrm{Ag}}{ }^{+} 222$ in $\mathrm{AN}$ reference). Formal potentials obtained in this study for cobaltocene may be referred to potentials for ferrocene measured in a number of protic and aprotic ionic liquids [34]. Table 2 presents differences between formal potentials of ferrocene/ferrocenium and cobaltocenium/cobaltocene couples in ionic liquids as well as molecular liquids; these results were calculated from the $1 / 2\left(E_{\mathrm{pa}}+E_{\mathrm{pc}}\right)$ values measured versus the cryptate electrode Ref. [34], or versus reference systems mentioned in references [15, 28-41]. Inspection of Table 2 shows that the $E_{1 / 2}\left(\mathrm{Fc} / \mathrm{Fc}^{+}\right)-E_{1 / 2}\left(\mathrm{Cc}^{+} / \mathrm{Cc}\right)$ difference may be approximated by the value $(1.350 \pm 0.020) \mathrm{V}$ and is in agreement with values obtained by other authors $[15,32,40,41]$. Such good agreement of the potential difference $( \pm 0.02 \mathrm{~V})$ suggests that the solvation of both reference couples is nearly independent of the electrolyte.

\subsection{Diffusion}

Diffusion coefficients of the cobaltocenium cation were calculated from the RandlesSevcik equation for a one-electron reduction:

$$
\left|j_{\mathrm{pc}}\right|=0.4463\left(\frac{F^{3}}{R T}\right)^{1 / 2}\left[\mathrm{Cc}^{+}\right] D^{1 / 2} v^{1 / 2}
$$

where $F$ is the Faraday constant, $R$ is the gas constant, $T$ is the temperature, $\left[\mathrm{Cc}^{+}\right]$is the cobaltocenium bulk concentration, $D$ stands for the $\mathrm{Cc}^{+}$diffusion coefficient, and $v$ is the sweep rate. Figure 2 presents examples of the cathodic peak current density as a function

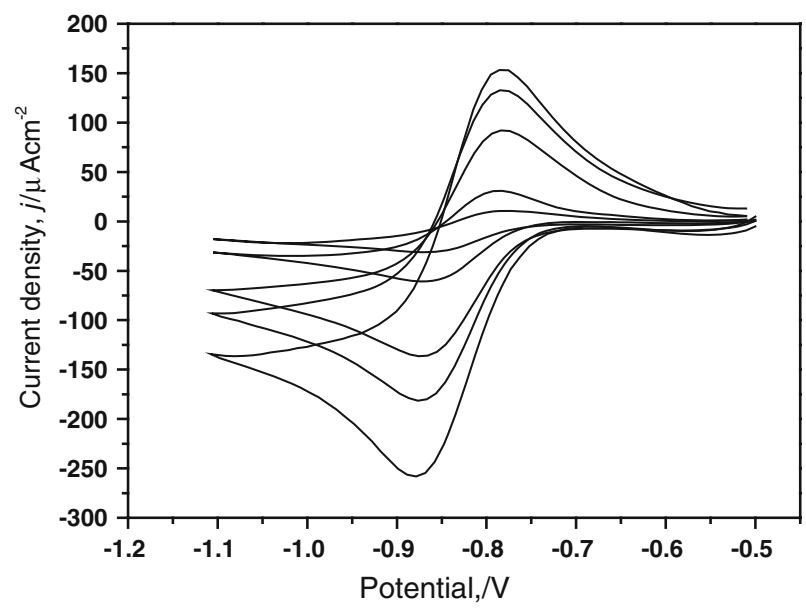

Fig. 1 Cyclic voltammetry for cobaltocene $\mathrm{Cc}^{+} / \mathrm{Cc}$ in BuMeImOTf on a Pt electrode at different sweep rates (from 2 to $200 \mathrm{mV} \mathrm{s}^{-1}$ ); reference electrode: $\mathrm{Ag} / \mathrm{Ag}^{+} 222$ in acetonitrile 
Table 1 Electrochemical parameters for cobaltocene cyclic voltammetry in aprotic ionic liquids at platinum, gold and glassy carbon electrodes, with reference electrode $\mathrm{AglAg}^{+}\left(0.01 \mathrm{~mol} \cdot \mathrm{L}^{-1}\right)$ cryptand $222+\left(0.1 \mathrm{~mol} \cdot \mathrm{L}^{-1}\right) \mathrm{AN}$

\begin{tabular}{|c|c|c|c|c|c|c|c|c|}
\hline Electrolyte/ & $\begin{array}{l}\text { Scan } \\
\text { rate } \\
\mathrm{mV} \cdot \mathrm{s}^{-1}\end{array}$ & $E_{\mathrm{pa}}$ & $E_{\mathrm{pc}}$ & $\Delta E_{p}$ & $1 / 2\left(E_{\mathrm{pa}}+E_{\mathrm{pc}}\right)$ & $\begin{array}{l}E_{p}-E_{p / 2} \\
\mathrm{~V}\end{array}$ & $\begin{array}{l}j_{\mathrm{pa}} \\
\mu \mathrm{A} \cdot \mathrm{cm}^{-2}\end{array}$ & $\begin{array}{l}j_{\mathrm{pc}} \\
\mu \mathrm{A} \cdot \mathrm{cm}^{-2}\end{array}$ \\
\hline $\mathrm{MePrPyrrNTf}_{2}$ & 2 & -0.752 & -0.864 & 0.112 & -0.808 & 0.067 & 44 & 40 \\
\hline $\mathrm{Pt}$ & 10 & -0.753 & -0.865 & 0.112 & -0.809 & 0.052 & 63 & 71 \\
\hline \multirow[t]{3}{*}{$\left(10.42 \mathrm{mmol} \cdot \mathrm{L}^{-1}\right)$} & 50 & -0.754 & -0.867 & 0.113 & -0.811 & 0.056 & 134 & 147 \\
\hline & 100 & -0.755 & -0.878 & 0.123 & -0.817 & 0.060 & 195 & 206 \\
\hline & 200 & -0.746 & -0.879 & 0.133 & -0.813 & 0.065 & 260 & 270 \\
\hline $\mathrm{MePrPyrrNTf}_{2}$ & 2 & -0.763 & -0.854 & 0.091 & -0.809 & 0.057 & 45 & 94 \\
\hline GC & 10 & -0.764 & -0.855 & 0.091 & -0.810 & 0.061 & 100 & 96 \\
\hline \multirow[t]{3}{*}{$\left(10.42 \mathrm{mmol} \cdot \mathrm{L}^{-1}\right)$} & 50 & -0.757 & -0.859 & 0.102 & -0.808 & 0.059 & 222 & 219 \\
\hline & 100 & -0.759 & -0.861 & 0.102 & -0.810 & 0.057 & 317 & 317 \\
\hline & 200 & -0.762 & -0.875 & 0.113 & -0.819 & 0.063 & 444 & 447 \\
\hline $\mathrm{MePrPyrrNTf}_{2}$ & 2 & -0.762 & -0.853 & 0.091 & -0.808 & 0.059 & 39 & 43 \\
\hline $\mathrm{Au}$ & 10 & -0.773 & -0.854 & 0.081 & -0.814 & 0.058 & 91 & 93 \\
\hline \multirow[t]{3}{*}{$\left(10.42 \mathrm{mmol} \cdot \mathrm{L}^{-1}\right)$} & 50 & -0.775 & -0.865 & 0.090 & -0.820 & 0.058 & 204 & 217 \\
\hline & 100 & -0.776 & -0.866 & 0.090 & -0.821 & 0.059 & 295 & 323 \\
\hline & 200 & -0.779 & -0.866 & 0.087 & -0.823 & 0.059 & 436 & 496 \\
\hline BuMeImOTf & 10 & -0.770 & -0.888 & 0.118 & -0.829 & 0.055 & 61 & 86 \\
\hline $\mathrm{Au}$ & 50 & -0.805 & -0.887 & 0.082 & -0.846 & 0.057 & 142 & 196 \\
\hline \multirow[t]{2}{*}{$\left(9.18 \mathrm{mmol} \cdot \mathrm{L}^{-1}\right)$} & 100 & -0.800 & -0.883 & 0.083 & -0.842 & 0.053 & 200 & 252 \\
\hline & 200 & -0.803 & -0.884 & 0.081 & -0.844 & 0.051 & 261 & 367 \\
\hline BuMeImOTf & 2 & -0.772 & -0.888 & 0.116 & -0.830 & 0.061 & 36 & 36 \\
\hline $\mathrm{GC}$ & 10 & -0.774 & -0.876 & 0.102 & -0.825 & 0.062 & 83 & 72 \\
\hline \multirow[t]{3}{*}{$\left(9.18 \mathrm{mmol} \cdot \mathrm{L}^{-1}\right)$} & 50 & -0.777 & -0.879 & 0.102 & -0.828 & 0.065 & 183 & 158 \\
\hline & 100 & -0.779 & -0.883 & 0.104 & -0.831 & 0.065 & 239 & 230 \\
\hline & 200 & -0.781 & -0.897 & 0.116 & -0.839 & 0.064 & 332 & 323 \\
\hline BuMeImOTf & 2 & -0.782 & -0.873 & 0.091 & -0.828 & 0.053 & 19 & 30 \\
\hline $\mathrm{Pt}$ & 10 & -0.783 & -0.874 & 0.091 & -0.829 & 0.063 & 66 & 63 \\
\hline \multirow[t]{3}{*}{$\left(9.18 \mathrm{mmol} \cdot \mathrm{L}^{-1}\right)$} & 50 & -0.784 & -0.876 & 0.092 & -0.830 & 0.059 & 135 & 131 \\
\hline & 100 & -0.785 & -0.877 & 0.092 & -0.831 & 0.057 & 182 & 184 \\
\hline & 200 & -0.785 & -0.878 & 0.093 & -0.832 & 0.054 & 242 & 267 \\
\hline BuMePyrrNTf $_{2}$ & 2 & -0.751 & -0.865 & 0.114 & -0.808 & 0.059 & 22 & 22 \\
\hline $\mathrm{Au}$ & 10 & -0.761 & -0.865 & 0.104 & -0.813 & 0.060 & 53 & 51 \\
\hline \multirow[t]{3}{*}{$\left(6.74 \mathrm{mmol} \cdot \mathrm{L}^{-1}\right)$} & 50 & -0.772 & -0.867 & 0.095 & -0.820 & 0.060 & 122 & 117 \\
\hline & 100 & -0.772 & -0.868 & 0.096 & -0.820 & 0.064 & 182 & 184 \\
\hline & 200 & -0.773 & -0.870 & 0.097 & -0.822 & 0.067 & 260 & 265 \\
\hline BuMePyrrNTf $_{2}$ & 2 & -0.752 & -0.843 & 0.091 & -0.798 & 0.060 & 21 & 22 \\
\hline $\mathrm{GC}$ & 10 & -0.753 & -0.844 & 0.091 & -0.799 & 0.060 & 49 & 49 \\
\hline \multirow[t]{3}{*}{$\left(6.74 \mathrm{mmol} \cdot \mathrm{L}^{-1}\right)$} & 50 & -0.754 & -0.845 & 0.091 & -0.800 & 0.060 & 104 & 105 \\
\hline & 100 & -0.755 & -0.856 & 0.101 & -0.806 & 0.057 & 148 & 143 \\
\hline & 200 & -0.756 & -0.857 & 0.101 & -0.807 & 0.066 & 192 & 198 \\
\hline BuMePyrrNTf $_{2}$ & 2 & -0.762 & -0.843 & 0.081 & -0.803 & 0.056 & 17 & 20 \\
\hline
\end{tabular}


Table 1 continued

\begin{tabular}{|c|c|c|c|c|c|c|c|c|}
\hline Electrolyte/ & $\begin{array}{l}\text { Scan } \\
\text { rate } \\
\mathrm{mV} \cdot \mathrm{s}^{-1}\end{array}$ & $E_{\mathrm{pa}}$ & $E_{\mathrm{pc}}$ & $\Delta E_{p}$ & $1 / 2\left(E_{\mathrm{pa}}+E_{\mathrm{pc}}\right)$ & $E_{p}-E_{p / 2}$ & $\begin{array}{l}j_{\mathrm{pa}} \\
\mu \mathrm{A} \cdot \mathrm{cm}^{-2}\end{array}$ & $\mu \mathrm{A} \cdot \mathrm{cm}^{-2}$ \\
\hline \multirow{4}{*}{$\begin{array}{l}\mathrm{Pt} \\
\left(6.74 \mathrm{mmol} \cdot \mathrm{L}^{-1}\right)\end{array}$} & 10 & -0.763 & -0.833 & 0.070 & -0.798 & 0.058 & 44 & 45 \\
\hline & 50 & -0.764 & -0.844 & 0.080 & -0.804 & 0.057 & 95 & 108 \\
\hline & 100 & -0.765 & -0.845 & 0.080 & -0.805 & 0.057 & 135 & 132 \\
\hline & 200 & -0.766 & -0.845 & 0.079 & -0.806 & 0.055 & 185 & 176 \\
\hline \multirow{4}{*}{$\begin{array}{l}\text { BuMePyrrOTf } \\
\mathrm{Au} \\
\left(10.8 \mathrm{mmol} \cdot \mathrm{L}^{-1}\right)\end{array}$} & 10 & -0.782 & -0.885 & 0.103 & -0.834 & 0.051 & 42 & 75 \\
\hline & 50 & -0.784 & -0.887 & 0.103 & -0.836 & 0.051 & 105 & 160 \\
\hline & 100 & -0.786 & -0.889 & 0.103 & -0.838 & 0.051 & 161 & 236 \\
\hline & 200 & -0.789 & -0.891 & 0.102 & -0.840 & 0.056 & 240 & 320 \\
\hline \multirow{5}{*}{$\begin{array}{l}\text { BuMePyrrOTf } \\
\text { GC } \\
\left(10.8 \mathrm{mmol} \cdot \mathrm{L}^{-1}\right)\end{array}$} & 2 & -0.784 & -0.864 & 0.080 & -0.824 & 0.057 & 28 & 27 \\
\hline & 10 & -0.785 & -0.886 & 0.101 & -0.836 & 0.058 & 64 & 59 \\
\hline & 50 & -0.779 & -0.880 & 0.101 & -0.830 & 0.066 & 140 & 135 \\
\hline & 100 & -0.781 & -0.895 & 0.114 & -0.838 & 0.067 & 190 & 207 \\
\hline & 200 & -0.778 & -0.902 & 0.124 & -0.840 & 0.068 & 329 & 361 \\
\hline \multirow{5}{*}{$\begin{array}{l}\text { BuMePyrrOTf } \\
\mathrm{Pt} \\
\left(10.8 \mathrm{mmol} \cdot \mathrm{L}^{-1}\right)\end{array}$} & 2 & -0.752 & -0.864 & 0.112 & -0.808 & 0.061 & 14 & 12 \\
\hline & 10 & -0.763 & -0.877 & 0.114 & -0.820 & 0.050 & 45 & 50 \\
\hline & 50 & -0.753 & -0.901 & 0.148 & -0.827 & 0.059 & 139 & 131 \\
\hline & 100 & -0.754 & -0.915 & 0.161 & -0.835 & 0.06 & 150 & 200 \\
\hline & 200 & -0.753 & -0.921 & 0.168 & -0.837 & 0.055 & 197 & 268 \\
\hline \multirow{5}{*}{$\begin{array}{l}\mathrm{Et}_{2} \mathrm{MeSNTf}_{2} \\
\mathrm{Au} \\
\left(10.21 \mathrm{mmol} \cdot \mathrm{L}^{-1}\right)\end{array}$} & 2 & -0.792 & -0.873 & 0.081 & -0.833 & 0.055 & 43 & 51 \\
\hline & 10 & -0.792 & -0.877 & 0.085 & -0.835 & 0.059 & 101 & 112 \\
\hline & 50 & -0.783 & -0.875 & 0.092 & -0.829 & 0.059 & 237 & 254 \\
\hline & 100 & -0.784 & -0.876 & 0.092 & -0.830 & 0.059 & 336 & 372 \\
\hline & 200 & -0.785 & -0.877 & 0.092 & -0.831 & 0.057 & 473 & 534 \\
\hline \multirow{5}{*}{$\begin{array}{l}\mathrm{Et}_{2} \mathrm{MeSNTf}_{2} \\
\mathrm{GC} \\
\left(10.21 \mathrm{mmol} \cdot \mathrm{L}^{-1}\right)\end{array}$} & 2 & -0.783 & -0.874 & 0.091 & -0.829 & 0.059 & 50 & 51 \\
\hline & 10 & -0.784 & -0.875 & 0.091 & -0.830 & 0.056 & 113 & 118 \\
\hline & 50 & -0.785 & -0.877 & 0.092 & -0.831 & 0.059 & 262 & 262 \\
\hline & 100 & -0.787 & -0.879 & 0.092 & -0.833 & 0.059 & 378 & 372 \\
\hline & 200 & -0.789 & -0.882 & 0.093 & -0.836 & 0.058 & 475 & 542 \\
\hline \multirow{5}{*}{$\begin{array}{l}\mathrm{Et}_{2} \mathrm{MeSNTf}_{2} \\
\mathrm{Pt} \\
\left(10.21 \mathrm{mmol} \cdot \mathrm{L}^{-1}\right)\end{array}$} & 2 & -0.782 & -0.873 & 0.091 & -0.828 & 0.061 & 50 & 51 \\
\hline & 10 & -0.783 & -0.874 & 0.091 & -0.829 & 0.057 & 93 & 95 \\
\hline & 50 & -0.784 & -0.875 & 0.091 & -0.830 & 0.056 & 196 & 207 \\
\hline & 100 & -0.785 & -0.876 & 0.091 & -0.831 & 0.056 & 313 & 347 \\
\hline & 200 & -0.786 & -0.877 & 0.091 & -0.832 & 0.055 & 370 & 412 \\
\hline \multirow{4}{*}{$\begin{array}{l}\mathrm{Et}_{3} \mathrm{SNTf}_{2} \\
\mathrm{Au} \\
\left(6.12 \mathrm{mmol} \cdot \mathrm{L}^{-1}\right)\end{array}$} & 10 & -0.784 & -0.87 & 0.086 & -0.827 & 0.054 & 84 & 105 \\
\hline & 50 & -0.784 & -0.87 & 0.086 & -0.827 & 0.057 & 200 & 229 \\
\hline & 100 & -0.785 & -0.87 & 0.085 & -0.828 & 0.057 & 309 & 347 \\
\hline & 200 & -0.785 & -0.869 & 0.084 & -0.827 & 0.057 & 433 & 475 \\
\hline \multirow{5}{*}{$\begin{array}{l}\mathrm{Et}_{3} \mathrm{SNTf}_{2} \\
\mathrm{GC} \\
\left.6.12 \mathrm{mmol} \cdot \mathrm{L}^{-1}\right)\end{array}$} & 2 & -0.772 & -0.863 & 0.091 & -0.818 & 0.060 & 46 & 42 \\
\hline & 10 & -0.773 & -0.864 & 0.091 & -0.819 & 0.057 & 98 & 102 \\
\hline & 50 & -0.774 & -0.866 & 0.092 & -0.820 & 0.057 & 216 & 218 \\
\hline & 100 & -0.776 & -0.867 & 0.091 & -0.822 & 0.059 & 319 & 319 \\
\hline & 200 & -0.777 & -0.869 & 0.092 & -0.823 & 0.060 & 441 & 459 \\
\hline
\end{tabular}


Table 1 continued

\begin{tabular}{|c|c|c|c|c|c|c|c|c|}
\hline Electrolyte/ & $\begin{array}{l}\text { Scan } \\
\text { rate } \\
\mathrm{mV} \cdot \mathrm{s}^{-1}\end{array}$ & $E_{\mathrm{pa}}$ & $E_{\mathrm{pc}}$ & $\Delta E_{p}$ & $\begin{array}{l}1 / 2\left(E_{\mathrm{pa}}+E_{\mathrm{pc}}\right) \\
\mathrm{V}\end{array}$ & $\begin{array}{l}E_{p}-E_{p / 2} \\
\mathrm{~V}\end{array}$ & $\begin{array}{l}j_{\mathrm{pa}} \\
\mu \mathrm{A} \cdot \mathrm{cm}^{-2}\end{array}$ & $\begin{array}{l}j_{\mathrm{pc}} \\
\mu \mathrm{A} \cdot \mathrm{cm}^{-2}\end{array}$ \\
\hline $\mathrm{Et}_{3} \mathrm{SNTf}_{2}$ & 2 & -0.773 & -0.862 & 0.089 & -0.818 & 0.063 & 41 & 41 \\
\hline $\mathrm{Pt}$ & 10 & -0.783 & -0.863 & 0.080 & -0.823 & 0.060 & 88 & 88 \\
\hline \multirow[t]{3}{*}{$\left.6.12 \mathrm{mmol} \cdot \mathrm{L}^{-1}\right)$} & 50 & -0.774 & -0.864 & 0.090 & -0.819 & 0.061 & 192 & 198 \\
\hline & 100 & -0.775 & -0.864 & 0.089 & -0.820 & 0.060 & 273 & 289 \\
\hline & 200 & -0.776 & -0.864 & 0.088 & -0.820 & 0.061 & 366 & 409 \\
\hline $\operatorname{EtMeImN}(\mathrm{CN})_{2}$ & 2 & -0.782 & -0.894 & 0.112 & -0.838 & 0.060 & 111 & 111 \\
\hline $\mathrm{Au}$ & 10 & -0.792 & -0.894 & 0.102 & -0.843 & 0.065 & 383 & 445 \\
\hline \multirow[t]{3}{*}{$\left(9.49 \mathrm{mmol} \cdot \mathrm{L}^{-1}\right)$} & 50 & -0.792 & -0.895 & 0.103 & -0.844 & 0.061 & 417 & 475 \\
\hline & 100 & -0.792 & -0.895 & 0.103 & -0.844 & 0.061 & 588 & 636 \\
\hline & 200 & -0.802 & -0.883 & 0.081 & -0.843 & 0.060 & 845 & 897 \\
\hline $\mathrm{EtMeImN}(\mathrm{CN})_{2}$ & 2 & -0.782 & -0.893 & 0.111 & -0.838 & 0.069 & 127 & 136 \\
\hline GC & 10 & -0.782 & -0.894 & 0.112 & -0.838 & 0.056 & 227 & 280 \\
\hline \multirow[t]{3}{*}{$\left(9.49 \mathrm{mmol} \cdot \mathrm{L}^{-1}\right)$} & 50 & -0.793 & -0.885 & 0.092 & -0.839 & 0.060 & 319 & 348 \\
\hline & 100 & -0.794 & -0.885 & 0.091 & -0.840 & 0.059 & 575 & 633 \\
\hline & 200 & -0.795 & -0.887 & 0.092 & -0.841 & 0.063 & 1002 & 987 \\
\hline $\operatorname{EtMeImN}(\mathrm{CN})_{2}$ & 2 & -0.792 & -0.883 & 0.091 & -0.838 & 0.072 & 107 & 99 \\
\hline $\mathrm{Pt}$ & 10 & -0.792 & -0.883 & 0.091 & -0.838 & 0.068 & 190 & 207 \\
\hline \multirow[t]{3}{*}{$\left(9.49 \mathrm{mmol} \cdot \mathrm{L}^{-1}\right)$} & 50 & -0.803 & -0.883 & 0.080 & -0.843 & 0.065 & 369 & 441 \\
\hline & 100 & -0.803 & -0.884 & 0.081 & -0.844 & 0.065 & 511 & 587 \\
\hline & 200 & -0.803 & -0.884 & 0.081 & -0.844 & 0.064 & 689 & 789 \\
\hline $\mathrm{MePrPipNTf}_{2}$ & 2 & -0.762 & -0.844 & 0.082 & -0.803 & 0.058 & 21 & 30 \\
\hline $\mathrm{Au}$ & 10 & -0.763 & -0.855 & 0.092 & -0.809 & 0.061 & 66 & 68 \\
\hline \multirow[t]{3}{*}{$\left(10.45 \mathrm{mmol} \cdot \mathrm{L}^{-1}\right)$} & 50 & -0.764 & -0.858 & 0.094 & -0.811 & 0.061 & 146 & 159 \\
\hline & 100 & -0.764 & -0.871 & 0.107 & -0.818 & 0.062 & 205 & 240 \\
\hline & 200 & -0.759 & -0.892 & 0.133 & -0.826 & 0.059 & 278 & 295 \\
\hline $\mathrm{MePrPipNTf}_{2}$ & 2 & -0.773 & -0.876 & 0.103 & -0.825 & 0.057 & 30 & 44 \\
\hline GC & 10 & -0.755 & -0.847 & 0.092 & -0.801 & 0.060 & 61 & 62 \\
\hline \multirow[t]{3}{*}{$\left(10.45 \mathrm{mmol} \cdot \mathrm{L}^{-1}\right)$} & 50 & -0.761 & -0.853 & 0.092 & -0.807 & 0.061 & 151 & 147 \\
\hline & 100 & -0.754 & -0.868 & 0.114 & -0.811 & 0.069 & 218 & 216 \\
\hline & 200 & -0.792 & -0.9 & 0.108 & -0.846 & 0.068 & 384 & 354 \\
\hline
\end{tabular}

of square root of the sweep rate. Such a linear $j_{\mathrm{pc}}=f(\sqrt{v})$ function was observed in all of the ionic liquids. Values of the cobaltocenium diffusion coefficients, collected in Table 3, show that in the case of viscous aprotic RTILs the diffusion coefficients are of the order of $\left(10^{-6}\right.$ or $\left.10^{-7}\right) \mathrm{cm}^{2} \cdot \mathrm{s}^{-1}$. Diffusion coefficients are between $0.52 \times 10^{-7} \mathrm{~cm}^{2} \cdot \mathrm{s}^{-1}$ and $5.2 \times 10^{-7} \mathrm{~cm}^{2} \cdot \mathrm{s}^{-1}$. Recently, three ionic liquids $\left(\right.$ EtMeImNTf $_{2}$, BuMePyrrNTf $_{2}$ and $\mathrm{BuMeImPF}_{6}$ ) were studied by cyclic voltammetry and rotating disc voltammetry [30]. The diffusion coefficient obtained here for BuMePyrrNTf $_{2}$ is somewhat lower, ca. $0.72 \times 10^{-7} \mathrm{~cm}^{2} \cdot \mathrm{s}^{-1}$, in comparison with $1.29 \times 10^{-7} \mathrm{~cm}^{2} \cdot \mathrm{s}^{-1}$ reported in Ref. [30]. Values reported for other RTILs are $D\left(\right.$ EtMeImNTf $\left._{2}\right)=3.27 \times 10^{-7} \mathrm{~cm}^{2} \cdot \mathrm{s}^{-1}$ and $D\left(\mathrm{BuMeImPF}_{6}\right)=3.73 \times 10^{-8} \mathrm{~cm}^{2} \cdot \mathrm{s}^{-1}$ [30]. Recently the diffusion coefficient of $\mathrm{Cc}^{+}$in 
Table 2 A comparison of formal potentials of cobaltocene and ferrocene in ionic liquids (this study and a previous paper or literature results)

\begin{tabular}{|c|c|c|}
\hline Electrolyte & Electrode $^{\mathrm{a}}$ & $E_{1 / 2}\left(\mathrm{Fc} / \mathrm{Fc}^{+}\right)-E_{1 / 2}\left(\mathrm{Cc}^{+} / \mathrm{Cc}\right) / \mathrm{V}$ \\
\hline $\mathrm{BuMeImBF}_{4}$ & $\mathrm{Au}, \mathrm{GC}$ & $1.345[32]$ \\
\hline $\mathrm{BuMeImPF}_{6}$ & $\mathrm{Au}, \mathrm{GC}$ & $1.345[32]$ \\
\hline $\mathrm{EtMeImBF}_{4}$ & $\mathrm{Au}$ & $1.339[31]$ \\
\hline $\mathrm{EtMeImBF}_{4}$ & $\mathrm{Pt}$ & $1.333[31]$ \\
\hline $\mathrm{EtMeImBF}_{4}$ & GC & $1.336[31]$ \\
\hline $\mathrm{MePrPyrrNTf}_{2}$ & $\mathrm{Pt}$ & 1.325 this study and [34] \\
\hline $\mathrm{MePrPyrrNTf}_{2}$ & GC & 1.327 this study and [34] \\
\hline BuMeImOTf & $\mathrm{Au}$ & 1.362 this study and [34] \\
\hline BuMePyrrNTf $_{2}$ & $\mathrm{Au}$ & 1.363 this study and [34] \\
\hline BuMePyrrNTf $_{2}$ & $\mathrm{Pt}$ & $1.333[15]$ \\
\hline BuMePyrrOTf & GC & 1.347 this study and [34] \\
\hline $\mathrm{Et}_{2} \mathrm{MeSNTf}_{2}$ & $\mathrm{Au}$ & 1.348 this study and [34] \\
\hline $\mathrm{Et}_{3} \mathrm{SNTf}_{2}$ & $\mathrm{Au}$ & 1.354 this study and [34] \\
\hline $\operatorname{EtMe} \operatorname{ImN}(\mathrm{CN})_{2}$ & GC & 1.332 this study and [34] \\
\hline $\operatorname{EtMeImN}(\mathrm{CN})_{2}$ & $\mathrm{Pt}$ & 1.355 this study and [34] \\
\hline $\mathrm{MePrPipNTf}_{2}$ & $\mathrm{Au}$ & 1.362 this study and [34] \\
\hline EtMeImNTf $_{2}$ & Au. Pt. GC & $1.330[28]$ \\
\hline $\mathrm{MeImSBuNTf}_{2}$ & Au. Pt. GC & $1.340[28]$ \\
\hline THF & $\mathrm{Pt}$ & $1.400[39]$ \\
\hline DCM & $\mathrm{Pt}$ & 1.390 [39], $1.351[40]$ \\
\hline $\mathrm{AN}$ & $\mathrm{Pt}$ & $1.390[39], 1.350[41]$ \\
\hline
\end{tabular}

$A N$ acetonitrile, $D C M$ dichloromethane, $T H F$ tetrahydrofurane

$\mathrm{BuMeImBF}_{4}$ and $\mathrm{BuMeImPF}_{6}$ was reported [32] to be slightly concentration sensitive. Values of $D(\mathrm{Fc})$ in aprotic RTILs are usually of the order of $\left(10^{-8}-10^{-7}\right) \mathrm{cm}^{2} \cdot \mathrm{s}^{-1}$, similar to $D\left(\mathrm{Cc}^{+}\right)$. Diffusion coefficients of the ferrocene/ferrocenium and cobaltocenium/cobaltocene couples have also been reported for allyl substited pyrrolidinium, piperidinium and morpholinium-based ionic liquids [33]. Diffusion coefficient values are in the same range of $\left(10^{-7}-10^{-6}\right) \mathrm{cm}^{2} \cdot \mathrm{s}^{-1}$, depending on IL's viscosity.

According to the Stokes-Einstein equation, Eq. 2, the main factor influencing the diffusion coefficient is the medium's viscosity, $\eta$,

$$
D \eta=\frac{k_{\mathrm{B}} T}{6 \pi r},
$$

where $k_{\mathrm{B}}$ is the Boltzmann constant and $r$ is the cobaltocenium radius.

Table 3 shows the $D \eta$ values calculated on the basis of literature data on ionic liquid viscosities published in references [5, 42-48], which correspond to the Walden product. Inspection of Table 3 suggests that the $D \eta$ product may be approximated by the value $(0.80 \pm 0.4) \times 10^{-7} \mathrm{~cm} \cdot \mathrm{g} \cdot \mathrm{s}^{-2}$. This result may also suggest that the cobaltocenium radius is constant and independent of the medium. An interesting aspect is a comparison (ratio) of diffusion coefficients of both popular metallocecenes, ferrocene and cobaltocenium, used as electrode potential references, $D(\mathrm{Fc}) / D\left(\mathrm{Cc}^{+}\right)$. Here, the solvation of both forms may be different due to the fact that ferrocene is a neutral molecule, while cobaltocenium is a cation. A comparison of $D\left(\mathrm{Cc}^{+}\right)$values (Table 3) with the corresponding $D(\mathrm{Fc})$ literature 


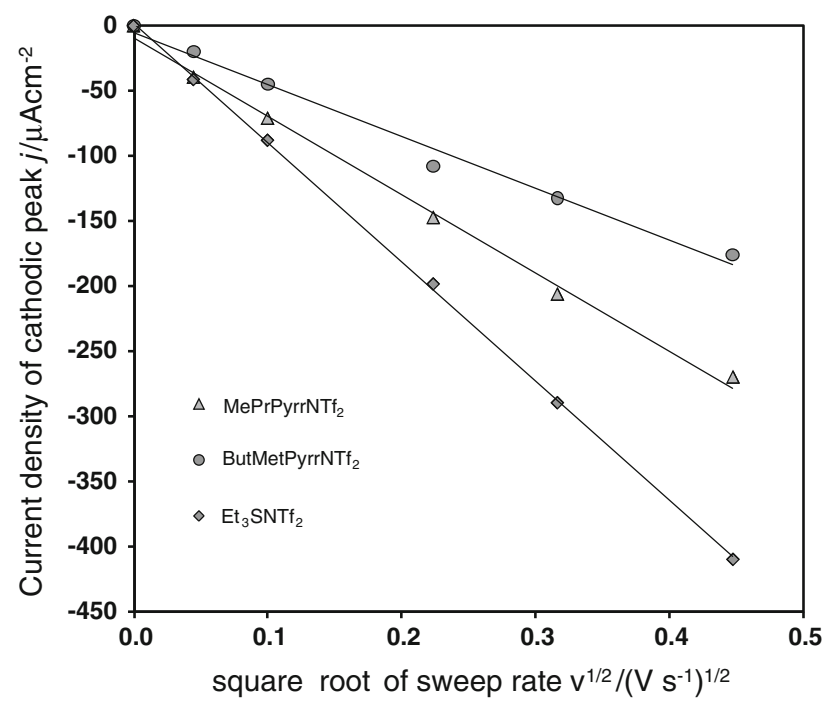

Fig. 2 Dependence of the cathodic current peak density on the square root of sweep rate for cobaltocene reduction in ionic liquids: black triangle, MePrPyrrNTf ${ }_{2}$; black circle, BuMePyrrNTf ${ }_{2}$; black diamond, $\mathrm{Et}_{3} \mathrm{SNTf}_{2}$

Table 3 Cobaltocenium $\left(\mathrm{Cc}^{+}\right)$diffusion coefficients $D\left(\mathrm{~cm}^{2} \mathrm{~s}^{-1}\right)$ in ionic liquids, the ionic liquid viscosity $\eta$ together with the $D \eta$ product

\begin{tabular}{lllll}
\hline Ionic liquid & Electrode & $D\left(\mathrm{Cc}^{+}\right) \mathrm{cm}^{2} \cdot \mathrm{s}^{-1} \times 10^{7}$ & $\eta \mathrm{cP}$ & $D \eta \mathrm{cm} \cdot \mathrm{g} \cdot \mathrm{s}^{-2} \times 10^{7}$ \\
\hline MePrPyrrNTf $_{2}$ & $\mathrm{Au}, \mathrm{GC}, \mathrm{Pt}$ & 1.09 & $59[42]$ & 0.64 \\
BuMeImOTf $_{\text {BuMePyrrNTf }}$ & $\mathrm{Au}, \mathrm{GC}, \mathrm{Pt}$ & 0.80 & $90[4]$ & 0.72 \\
BuMePyrrOTf $_{\text {Et }}$ & $\mathrm{Au}, \mathrm{GC}, \mathrm{Pt}$ & 0.72 & $85[43]$ & 0.61 \\
Et $_{2} \mathrm{MeSNTf}_{2}$ & $\mathrm{Au}, \mathrm{GC}, \mathrm{Pt}$ & 0.52 & $158[48]$ & 0.82 \\
Et $_{3} \mathrm{SNTf}_{2}$ & $\mathrm{Au}, \mathrm{GC}, \mathrm{Pt}$ & 1.64 & $36[5]$ & 0.59 \\
EtMeImN $_{2}(\mathrm{CN})_{2}$ & $\mathrm{Au}, \mathrm{GC}, \mathrm{Pt}$ & 3.74 & $33[47]$ & 1.23 \\
$\mathrm{MePrPipNTf}_{2}$ & $\mathrm{Au}, \mathrm{GC}, \mathrm{Pt}$ & 0.58 & $21[44]$ & 1.09 \\
\hline
\end{tabular}

values shows that $D(\mathrm{Fc}) / D\left(\mathrm{Cc}^{+}\right)>1$. For example, according to Ref. [31], the $D(\mathrm{Fc}) /$ $D\left(\mathrm{Cc}^{+}\right)$ratio is 3.0 in $\mathrm{EMImBF}_{4}\left\{D(\mathrm{Fc})=2.70 \times 10^{-7} \mathrm{~cm}^{2} \cdot \mathrm{s}^{-1}\right\}$, while the corresponding value in $\mathrm{EMImNTf}_{2}$, reported in Ref. [30] is $1.39\left\{D(\mathrm{Fc})=3.27 \times 10^{-7}\right.$ $\left.\mathrm{cm}^{2} \cdot \mathrm{s}^{-1}\right\}$. For comparison, the $D(\mathrm{Fc}) / D\left(\mathrm{Cc}^{+}\right)$ratio is also higher than unity in solutions with molecular solvents. For example $D(\mathrm{Fc}) / D\left(\mathrm{Cc}^{+}\right)=1.77$ in acetonitrile $\left\{D(\mathrm{Fc})=2.3 \times 10^{-5} \mathrm{~cm}^{2} \cdot \mathrm{s}^{-1}[49]\right.$ and $D\left(\mathrm{Cc}^{+}\right)=1.30 \times 10^{-5} \mathrm{~cm}^{2} \cdot \mathrm{s}^{-1}$ [39] $\}$. All of this information suggests that the $\mathrm{Fc}$ molecule and $\mathrm{Cc}^{+}$cation, although of similar shape and radius, are probably solvated differently, as suggested in Ref. [50]. The cobaltocenium cation may interact with anions present in the electrolyte and therefore have a higher effective radius and, hence, a somewhat lower diffusion coefficient. In general, diffusion coefficients of large organic compounds determined in RTILs are on the order of $10^{-7} \mathrm{~cm}^{2} \cdot \mathrm{s}^{-1}$ which is two orders of magnitude lower than in conventional molecular solvents [51]. 


\section{Conclusions}

1. Well-defined, diffusion controlled, anodic and cathodic peaks were found for the $\mathrm{Cc}^{+} /$ Cc redox couple in a number of RTILs

2. Values of $E_{\mathrm{p}}-E_{\mathrm{p} / 2}$ are close to $56 \mathrm{mV}$, typical of reversible processes. On the other hand, the difference between cathodic and anodic peak potentials, $E_{\mathrm{pa}}-E_{\mathrm{pc}}$, is $\geq 81 \mathrm{mV}$, while the value predicted by theory for reversible processes is $57-60 \mathrm{mV}$, which indicates a quasi-reversible redox process

3. The $E_{1 / 2}\left(\mathrm{Fc} / \mathrm{Fc}^{+}\right)-E_{1 / 2}\left(\mathrm{Cc}^{+} / \mathrm{Cc}\right)$ difference may be approximated by the value $(1.350 \pm 0.020) \mathrm{V}$

4. Values of the cobaltocenium $\left(\mathrm{Cc}^{+}\right)$diffusion coefficients $D$ are in the range of $0.5 \times 10^{-7} \mathrm{~cm}^{2} \cdot \mathrm{s}^{-1}-5.2 \times 10^{-7} \mathrm{~cm}^{2} \cdot \mathrm{s}^{-1}$, depending on the medium's viscosity $\eta$. The $D \eta$ value, corresponding to the Walden product, falls within the narrow range of $0.61 \times 10^{-7} \mathrm{~cm} \cdot \mathrm{g} \cdot \mathrm{s}^{-2}-1.23 \times 10^{-7} \mathrm{~cm} \cdot \mathrm{g} \cdot \mathrm{s}^{-2}$

Acknowledgments Support of grant BW31-208/10 is gratefully acknowledged.

Open Access This article is distributed under the terms of the Creative Commons Attribution License which permits any use, distribution, and reproduction in any medium, provided the original author(s) and the source are credited.

\section{References}

1. Earle, M.J., Seddon, K.R.: Ionic liquids. Green solvents for the future. Pure Appl. Chem. 72, 1391-1398 (2000)

2. Wasserschied, P., Keim, W.: Ionic liquids-New solutions for transition metal catalysis. Angew. Chem. Int. Ed. 39, 3772-3789 (2000)

3. Zhang, S., Sun, N., He, X., Lu, X., Zhang, X.: Physical properties of ionic liquids: database and evaluation. J. Phys. Chem. Ref. Data 35, 1475-1517 (2006)

4. Bonhote, P., Dias, A.P., Papageorgiou, N., Kalyanasundaram, K., Greatzel, M.: Hydrophobic, highly conductive ambient-temperature molten salts. Inorg. Chem. 35, 1168-1178 (1996)

5. Fang, S., Yang, L., Wei, C., Peng, C., Tachibana, K., Kamijima, K.: Low-viscosity and low-melting point asymmetric trialkylsulfonium based ionic liquids as potential electrolytes. Electrochem. Commun. 9, 2696-2702 (2007)

6. MacFarlane, D.R., Meakin, P., Sun, J., Amini, N., Forsyth, M.: Pyrrolidinium imides: a new family of molten salts and conductive plastic crystal phases. J. Phys. Chem. B 106, 4146-4170 (1999)

7. Ohno, H.: Electrochemical Aspects of Ionic Liquids. John Wiley Inc., New Jersey (2005)

8. Galinski, M., Lewandowski, A., Stępniak, I.: Ionic liquids as electrolytes. Electrochim. Acta 51, 5567-5580 (2006)

9. Silvester, D.S., Compton, R.G.: Electrochemistry in room temperature ionic liquids: a review and some possible applications. Z. Phys. Chem. 220, 1247-1274 (2006)

10. Zhao, C., Burrel, G., Torriero, A.A.J., Separovic, F., Dunlop, N.F., MacFarlane, D.R., Bond, A.M.: Electrochemistry of room temperature protic ionic liquids. J. Phys. Chem. B 112, 6923-6936 (2008)

11. Suarez, P.A.Z., Selbach, V.M., Dullius, J.E.L., Einloft, S., Piatnicki, C.M.S., Azambuja, D.S., de Souza, R.F., Dupont, J.: Enlarged electrochemical window in dialkyl-imidazolium cation based room-temperature air and molten salts. Electrochim. Acta 42, 2533-2535 (1997)

12. Evans, R.G., Klymenko, O.V., Hardacre, C., Seddon, K.R., Compton, R.G.: Oxidation of N, N, N', N'tetraalkyl-para-phenylenediamines in a series of room temperature ionic liquids incorporating the bis(trifluoromethylsulfonyl)imide anion. J. Electroanal. Chem. 556, 179-188 (2003)

13. Fukui, R., Katayama, Y., Miura, T.: Electrodeposition of cobalt from hydrophobic room-temperature molten salt system. Electrochemistry 73, 567-569 (2005)

14. Saheb, A., Janata, J., Josowicz, M.: Reference electrode for ionic liquids. Electroanalysis 18, 405-409 (2006) 
15. Snook, G.A., Best, A.S., Pandolfo, A., Hollenkamp, A.F.: Evaluation of a $\mathrm{AglAg}^{+}$reference electrode for use in room temperature ionic liquids. Electrochem. Commun. 8, 1405-1411 (2006)

16. Yasuda, K., Nohira, T., Ogata, Y.H., Ito, Y.: Electrochemical window of molten LiCl-KCl-CaCl 2 and the $\mathrm{Ag}^{+} / \mathrm{Ag}$ reference electrode. Electrochim. Acta 51, 561-565 (2005)

17. Lewandowski, A., Szukalska, A., Galinski, M.: A reference electrode for aprotic media based on a silver(I) complex with cryptand 222. New J. Chem. 19, 1259-1263 (1995)

18. Lewandowski, A., Osinska, M., Swiderska-Mocek, A., Galinski, M.: Cryptate reference electrode for ionic liquids. Electroanalysis 20, 1903-1908 (2008)

19. Rogers, E.I., Silvester, D.L., Poole, D.L., Aldous, L., Hardacre, C., Compton, R.G.: Voltammetric characterization of the ferrocenelferrocenium and cobaltoceniumlcobaltocene redox couples in RTILs. J. Phys. Chem. C 112, 2729-2735 (2008)

20. Eisele, S., Schwartz, M., Speiser, B., Tittle, C.: Diffusion coefficient of ferrocene in 1-butyl-3-methylimidazolium tetrafluoroborate-Concentration dependence and solvent purity. Electrochim. Acta 51, 5304-5306 (2006)

21. Nagy, L., Gyetvai, G., Kollar, L., Nagy, G.: Electrochemical behavior of ferrocene in ionic liquid media. J. Biochem. Biophys. Meth. 69, 121-132 (2006)

22. Hultgren, V., Mariotti, A.W.A., Bond, A.M., Wedd, A.G.: Reference potential calibration and voltammetry at macrodisk electrodes of metallocene derivatives in the ionic liquid [bmim][PF 6 . Anal. Chem. 74, 3151-3156 (2002)

23. Brooks, C., Doherty, A.P.: Concentration-dependent diffusion in room temperature ionic liquids: a microelectrode study. Electrochem. Commun. 6, 867-871 (2004)

24. Matsumiya, M., Terazone, M., Tokuraku, K.: Temperature dependence of kinetics and diffusion coefficients for ferrocene/ferricenium in ammonium-imide ionic liquids. Electrochim. Acta 51, $1178-1183$ (2006)

25. Fietkau, N., Clegg, A.D., Evans, R.G., Villagran, C., Hardacre, C., Compton, R.G.: Electrochemical rate constants in room temperature ionic liquids: the oxidation of a series of ferrocene derivatives. ChemPhysChem 7, 1041-1045 (2006)

26. Matsumiya, M., Suda, S., Tsunashima, K., Sugiya, M., Kishioka, S., Matsuura, H.: Electrochemical behaviors of multivalent complexes in room temperature ionic liquids based on quaternary phosphonium cations. J. Electroanal. Chem. 622, 129-135 (2008)

27. Wang, H., Zhao, C., Bhatt, A.I., MacFarlane, D.R., Lu, J.X., Bond, A.M.: Electrochemical study of Dialcarb "distillable" room-temperature ionic liquids. ChemPhysChem 10, 455-461 (2009)

28. Torriero, A.A.J., Siriwardana, A.I., Bond, A.M., Burgar, I.M., Dunlop, N.F., Deacon, G.B., MacFarlane, D.R.: Physical and electrochemical properties of thioether-functionalized ionic liquids. J. Phys. Chem. B 113, 11222-11231 (2009)

29. Zhang, J., Bond, A.M.: Practical considerations associated with voltammetric studies in room temperature ionic liquids. Analyst 130, 1132-1147 (2005)

30. Sukardi, S.K., Zhang, J., Burgar, I., Horne, M.D., Hollenkamp, A.F., MacFarlane, D.R., Bond, A.M.: Prospects for a widely applicable reference potential scale in ionic liquids based on ideal reversible reduction of the cobaltocenium cation. Electrochem. Commun. 10, 250-254 (2008)

31. Waligora, L., Lewandowski, A., Gritzner, G.: Electrochemical studies of four organometallic redox couples as possible reference redox systems in 1-ethyl-3-methylimidazolium tetrafluoroborate. Electrochim. Acta 54, 1414-1419 (2009)

32. Shiddiky, M.J.A., Torriero, A.J., Zhao, C., Burgar, I., Kennedy, G., Bond, A.M.: Nonadditivity of faradaic currents and modification of capacitance currents in the voltammetry of mixtures of ferrocene and the cobaltocenium cation in protic and aprotic Ionic liquids. J. Am. Chem. Soc. 131, 7976-7989 (2009)

33. Wu, T.Y., Su, S.G., Wang, H.P., Lin, Y.C., Gung, S.T., Lin, M.W., Sun, I.W.: Electrochemical studies and self diffusion coefficients in cyclic ammonium based ionic liquids with allyl substituents. Electrochim. Acta 56, 3209-3218 (2011)

34. Lewandowski, A., Waligora, L., Galinski, M.: Ferrocene as a reference redox couple for aprotic ionic liquids. Electroanalysis 21, 2221-2227 (2009)

35. MacFarlane, D.R., Golding, J., Forsyth, S., Forsyth, M., Deacon, G.B.: Low viscosity ionic liquids based on organic salts of the dicyanamide anion. Chem. Commun. 2001, 1430-1431 (2001)

36. Lipinska, K.: Preparations of Dicyanamide Based Ionic Liquids. MS Thesis, Poznan University of Technology (2007)

37. Zhou, Z.B., Matsumoto, H., Tatsumi, K.: Cyclic quaternary ammonium ionic liquids with perfluoroalkyltrifluoroborates: synthesis, characterization, and properties. Chem. Eur. J. 12, 2196-2212 (2006) 
38. Gosser, D.K. Jr.: Cyclic Voltammetry: Simulation and Analysis of Reaction Mechanisms. VCH Publishers (1993)

39. Aranzaes, J.R., Daniel, M.-C., Astruc, D.: Metallocenes as references for the determination of redox potentials by cyclic voltammetry-Permethylated iron and cobalt sandwich complexes, inhibition by polyamine dendrimers, and the role of hydroxy-containing ferrocenes. Can. J. Chem. 84, 288-299 (2006)

40. Tsierkezos, N.G.: Electron transfer kinetics for the cobaltocene $(+1 / 0)$ couple at platinum disk electrode in acetonitrile/dichloromethane binary solvent system. J. Mol. Liq. 138, 1-8 (2008)

41. Stojanovic, R.S., Bond, A.M.: Examination of conditions under which the reduction of the cobaltocenium cation can be used as a standard voltammetric reference process in organic and aqueous solvents. Anal. Chem. 65, 56-64 (1993)

42. Yim, T., Lee, H.Y., Kim, H.J., Mun, J., Kim, S., Oh, S.M., Kim, Y.G.: Synthesis and properties of pyrrolidinium and piperidinium bis(trifluoromethanesulfonyl)imide ionic liquids with allyl substituents. Bull. Korean Chem. Soc. 28, 1567-1572 (2008)

43. McFarlane, D.R., Sun, J., Golding, J., Meakin, P., Forsyth, M.: High conductivity molten salts based on the imide ion. Electrochim. Acta 45, 1271-1278 (2000)

44. MacFarlane, D.R., Forsyth, S.A., Golding, J., Deacon, G.B.: Ionic liquids based on imidazolium, ammonium and pyrrolidinium salts of the dicyanamide anion. Green Chem. 4, 444-448 (2002)

45. Olivier-Bourbigou, H., Magna, L.: Ionic liquids: perspectives for organic and catalytic reactions. J. Mol. Catal. A: Chem. 182-183, 419-437 (2002)

46. Greaves, T.L., Weerawardena, A., Fong, C., Krodkiewska, I., Drummond, C.J.: Protic ionic liquids: solvents with tunable phase behavior and physicochemical properties. J. Phys. Chem. B 110, 22479-22487 (2006)

47. Matsumoto, H., Sakaebe, H., Tatsumi, K.: Preparation of room temperature ionic liquids based on aliphatic onium cations and asymmetric amide anions and their electrochemical properties as a lithium battery electrolyte. J. Power Sources 146, 45-50 (2005)

48. Boso, M., Mastragostino, M., Montanino, M., Passerini, S., Soavi, F.: Electropolymerization of poly(3methylthiophene) in pyrrolidinium-based ionic liquids for hybrid supercapacitors. Electrochim. Acta 53, 7967-7971 (2008)

49. Clegg, A.D., Rees, N.V., Klymenko, O.V., Coles, B.A., Compton, R.G.: Marcus theory of outer-sphere heterogeneous electron transfer reactions: high precision steady-state measurements of the standard electrochemical rate constant for ferrocene derivatives in alkyl cyanide solvents. J. Electroanal. Chem. 580, 78-86 (2005)

50. Kuznetsov, A.M., Maslii, A.N., Krishtalik, L.I.: Solvation of ferrocene, cobaltocene, and their ions by the data of quantum-chemical calculations. Russ. J. Electrochem. 45, 93-98 (2009)

51. Hapiot, P., Lagrost, C.: Electrochemical reactivity in room-temperature ionic liquids. Chem. Rev. 108, 2238-2264 (2008) 